\title{
Impact of age, sex and body mass index on cortisol secretion in 143 healthy adults
}

\author{
Ferdinand Roelfsema', Diana van Heemst² , Ali Iranmanesh ${ }^{3}$, Paul Takahashi ${ }^{4}$, \\ Rebecca Yang ${ }^{5}$ and Johannes D Veldhuis ${ }^{5}$
}

\author{
${ }^{1}$ Department of Internal Medicine, Leiden University Medical Center, Leiden, The Netherlands \\ 2Department of Internal Medicine, Section Gerontology and Geriatrics, Leiden University Medical Center, \\ Leiden, The Netherlands \\ ${ }^{3}$ Endocrine Section, Medical Service, Salem Veterans Affairs Medical Center, Salem, Virginia, USA \\ ${ }^{4}$ Primary Care Internal Medicine, Mayo Clinic, Rochester, Minnesota, USA \\ ${ }^{5}$ Endocrine Research Unit, Mayo Medical and Graduate Schools, Clinical Translational Research Center, Mayo Clinic, \\ Rochester, Minnesota, USA
}

\begin{abstract}
Context: Studies on 24-h cortisol secretion are rare. The impact of sex, age and adiposity on cortisol levels, often restricted to one or a few samples, are well recognized, but conflicting.

Objective: To investigate cortisol dynamics in 143 healthy men and women, spanning 7 decades and with a 2-fold body mass index (BMI) range with different analytic tools. Setting: Clinical Research Unit.

Design: Cortisol concentrations in 10-min samples collected for $24 \mathrm{~h}$. Outcomes were mean levels, deconvolution parameters, approximate entropy (ApEn, regularity statistic) and 24-h rhythms.

Results: Total 24-h cortisol secretion rates estimated by deconvolution analysis were sex, age and BMI independent. Mean 24-h cortisol concentrations were lower in premenopausal women than those in men of comparable age (176 $\pm 8.2 \mathrm{vs}$ $217 \pm 9.4 \mathrm{nmol} / \mathrm{L}, P=0.02$ ), but not in subjects older than 50 years. This was due to lower daytime levels in women, albeit similar in the quiescent overnight period. Aging increased mean cortisol by $10 \mathrm{nmol} / \mathrm{L}$ per decade during the quiescent secretory phase and advanced the acrophase of the diurnal rhythm by $24 \mathrm{~min} /$ decade. However, total 24-h cortisol secretion rates estimated by deconvolution analysis were sex, age and BMI independent. ApEn of 24-h profiles was higher (more random) in premenopausal women than those in men $(1.048 \pm 0.025$ vs $0.933 \pm 0.023, P=0.001)$, but not in subjects older than 50 years. ApEn peaked during the daytime.

Conclusion: Sex and age jointly determine the 24-h cortisol secretory profile. Sex effects are largely restricted to age $<50$ years, whereas age effects elevate concentrations in the late evening and early night and advance the timing of the peak diurnal rhythm.
\end{abstract}

Correspondence should be addressed to J D Veldhuis Email veldhuis.johannes@mayo.edu
Endocrine Connections (2017) 6, 500-509

\section{Introduction}

The hypothalamic-pituitary-adrenal (HPA) axis is the most important neuroendocrine stress-responsive system, which is of critical importance for survival in mammals (1).
Tightly controlled regulation of the HPA axis is crucial for maintaining both mental and physical health, in as much as hypoactivity and hyperactivity of this system leads to

$\begin{array}{lr}\text { http://www.endocrineconnections.org } & \text { ○ } 2017 \text { The authors } \\ \text { DOI: } 10.1530 / \text { EC-17-0160 } & \text { Published by Bioscientifica Ltd }\end{array}$

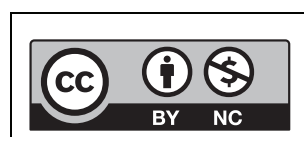

This work is licensed under a Creative Commons Attribution-NonCommercial 4.0 International License. 
disease states $(2,3,4,5,6,7,8,9)$. Although the HPA axis has been studied for several decades, published reports on the influence of age, sex and body mass on HPA dynamics are conflicting $(3,4,5,6,10,11,12,13,14,15,16,17$, 18). The small size of most cohorts evaluated to date, the narrow age and BMI ranges encompassed and the lack of inclusion of both genders collectively make the possibility of statistical type I or type II errors high in earlier studies. As importantly, because of correlations among age, BMI and gender, multivariate regression is needed for definitive inferences. Nonetheless, multivariate analysis also is unreliable in small cohorts $(19,20)$. In addition, given the large concentration difference across the 24-h cycle and the marked pulsatility of cortisol, studies using a single sample, or a few blood samples, have large random variance, and are not representative of finely timed physiologically regulated cortisol secretion per se. To overcome these obstacles would require investigation of a larger number of healthy unmedicated adults, both men and women, over wide ranges of age and BMI, and utilization of suitable analytical tools to quantify the dependencies of dynamic cortisol secretion properties (mean, peak, nadir, acrophase, basal and pulsatile, 24-h secretion, pattern regularity or ApEn) on individual and/ or combined clinical characteristics. The present analyses seek to address these issues in 143 healthy individuals sampled frequently (every $10 \mathrm{~min}$ ) for a sufficiently representative duration $(24 \mathrm{~h})$ and analyzed with robust immunoassays.

\section{Subjects and methods}

\section{Clinical protocol}

The 109 volunteers from Leiden, The Netherlands, had participated as controls in studies on neurological disorders, obesity, longevity and ACTH-cortisol relationships (3, 4, $9,21)$. In addition, 34 healthy male participants of a not yet published sleep interference study were included from Rochester, MN, USA. Informed consent was obtained from each subject after full explanation of the purpose and nature of all procedures used as approved by the Mayo Clinic Internal Review Board and Leiden University Ethics Committee. Clinical characteristics are listed in Table 1. Postmenopausal individuals studied here did not use estrogen therapy. Premenopausal women were studied in the early follicular phase of the menstrual cycle. Participants maintained conventional work and sleeping patterns and reported no recent transmeridian travel, weight change ( $>2 \mathrm{~kg}$ in 6 week), shift work,
Table 1 Demographic properties of the volunteers.

\begin{tabular}{|c|c|c|c|}
\hline & Men (79) & Women (64) & $P$ value \\
\hline Age (year) & $47.2 \pm 1.9$ & $45.4 \pm 1.7$ & 0.93 \\
\hline BMI $\left(\mathrm{kg} / \mathrm{m}^{2}\right)$ & $26.8 \pm 0.6$ & $27.9 \pm 0.7$ & 0.28 \\
\hline IGF-1 (nmol/L) & $19.9 \pm 0.7$ & $19.4 \pm 0.8$ & 0.62 \\
\hline Free T4 (nmol/L) & $15.8 \pm 0.4$ & $15.0 \pm 0.2$ & 0.09 \\
\hline
\end{tabular}

Data are shown as mean and S.E.M. Statistical comparisons were done with the 2-tailed Student's $t$-test.

psychosocial stress, prescription medication use, including contraceptive pills in women, substance abuse, neuropsychiatric illness or acute or chronic systemic disease. A complete medical history, physical examination and screening biochemistry tests were normal. No subject had been exposed to glucocorticoids within the preceding 3 months. Volunteers were admitted to the Study Unit in the evening or stayed overnight in a nearby hotel (3). Ambulation was permitted to the lavatory only. Vigorous exercise, daytime sleep, snacks, caffeinated beverages and cigarette smoking were disallowed. Meals were provided at 09:00, 12:30 and 17:30 h, and room lights were turned off between 22:00 and 24:00h. Blood samples $(2.0 \mathrm{~mL})$ were withdrawn at 10 -min intervals for $24 \mathrm{~h}$. Total blood loss was less than $430 \mathrm{~mL}$. Volunteers were compensated for the time spent in the study as approved by the IRB.

\section{Assays}

Serum cortisol concentrations were measured with different assays. In the majority of the Leiden studies, cortisol was assayed by solid-phase RIA (Sorin Biomedical, Milan, Italy). Intra-assay and interassay CVs were 5.1 and $6.4 \%$, respectively. Cortisol in the Leiden Longevity Study was measured by the electrochemiluminescence immunoassay on the Modular E-170 immunoanalyzer (Roche Diagnostics). The detection limit of the first generation of the assay was $5.5 \mathrm{nmol} / \mathrm{L}$, and intra- and interassay CVs were $3.5-6.3 \%$ and $3.1-5.1 \%$, respectively. The Mayo study used Immulite 2000 (Siemens Healthcare Diagnostics, Flanders, NJ, USA). This chemiluminescent assay has a detection range of $5.5-1380 \mathrm{nmol} / \mathrm{L}$ with intra- and interassay coefficients of variation of $7.2-9.4 \%$ and $6.3-9.4 \%$ at respective concentrations of $10.5-122$ and $10.2-113 \mathrm{nmol} / \mathrm{L}$. All samples of each individual were measured the same assay. No samples were undetectable in any assay.

\section{Average, peak and nadir GH cortisol concentrations}

Mean (24-h and 3-h averages), median, absolute peak (single daily maximum) and absolute nadir (single daily

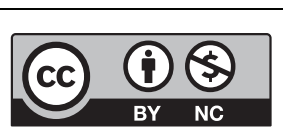

This work is licensed under a Creative Commons Attribution-NonCommercial 4.0 International License. 
minimum) cortisol concentrations were determined in each subject.

\section{Deconvolution analysis}

Cortisol concentration time series were analyzed using a recently developed automated deconvolution method. Pulse detection was empirically validated using hypothalamo pituitary sampling and simulated pulsatile time series. Sensitivity and specificity both exceed 93\%. The Matlab-based algorithm first detrends the data and normalizes concentrations to the unit interval $(0,1)$. Second, the program creates multiple successively decremental potential pulse-onset time sets, each containing one fewer pulse by a smoothing process (a nonlinear adaptation of the heat-diffusion equation). Third, a maximum-likelihood expectation (MLE) estimation method calculates all secretion and elimination parameters simultaneously conditional on each of the candidate pulse-time sets. Deconvolution parameters comprise basal secretion, secretory-burst mass (concentration units), mode (time delay in min to maximum value) and frequency (number of bursts per sampling duration, lambda of Weibull distribution). Pulsatile secretion is the sum of secretory-burst mass and total secretion of the sum of basal and pulsatile secretion. The fast half-life of cortisol was represented as $2.41 \mathrm{~min}$, constituting $37 \%$ of the decay amplitude and the slow half-life ranging between 40 and $70 \mathrm{~min}$. Statistical model selection was performed using the Akaike information criterion $(22,23)$.

\section{Approximate entropy}

Adaptive ensemble (network-like) control of hormone release patterns was appraised via the approximate entropy statistic (ApEn) (24). ApEn provides a model-free and scale-invariant statistical estimate of relative randomness in the data, wherein larger values denote greater process randomness and less feedback control $(25,26)$.

\section{Diurnal variations}

Diurnal variations of cortisol levels were studied in two ways: first, by relating mean concentrations in successive 3-h windows to age, BMI and sex; and secondly, by Cosinor analysis, which quantitates the nyctohemeral variation of cortisol concentrations by using a nonlinear unweighted least-squares cosine regression. Ninety-five
Table 2 Mean cortisol concentrations in 3-h bins in 143 healthy men and women.

\begin{tabular}{|c|c|c|c|}
\hline Time bin (h) & Women (64) & Men (79) & $P$ value \\
\hline $9-12$ & $240 \pm 11$ & $266 \pm 10$ & 0.09 \\
\hline $12-15$ & $190 \pm 8$ & $224 \pm 9$ & 0.008 \\
\hline $15-18$ & $154 \pm 8$ & $200 \pm 9$ & $<0.0001$ \\
\hline $18-21$ & $118 \pm 10$ & $137 \pm 11$ & 0.22 \\
\hline $21-24$ & $104 \pm 10$ & $99 \pm 8$ & 0.64 \\
\hline $24-3$ & $117 \pm 10$ & $122 \pm 7$ & 0.72 \\
\hline $3-6$ & $232 \pm 14$ & $244 \pm 9$ & 0.50 \\
\hline $6-9$ & $307 \pm 11$ & $352 \pm 13$ & 0.01 \\
\hline
\end{tabular}

Statistical calculations with the two-sided $t$-test were done on un transformed data. Data are mean \pm s.E.M. Time bins correspond to clock hours.

percent statistical confidence intervals were determined for the 24 -h cosineamplitude (50\% of the zenith-nadir difference), mesor (rhythmic mean) and acrophase (clock time of maximal value).

\section{Statistical analysis}

The primary endpoint was the 24-h mean cortisol concentration with segmentation into 3-h bins across the $24 \mathrm{~h}$. Secondary mechanistic outcomes were (1) deconvolution, (2) ApEn and (3) Cosinor parameters.

The a priori clinical hypothesis was that sex, age and/or BMI determine time-dependent cortisol concentrations. Comparisons between sexes were done with the Student's $t$-test for unpaired data. Data were analyzed using a General Linear Model (GLM) for repeat measurements with categorical variables, sex and

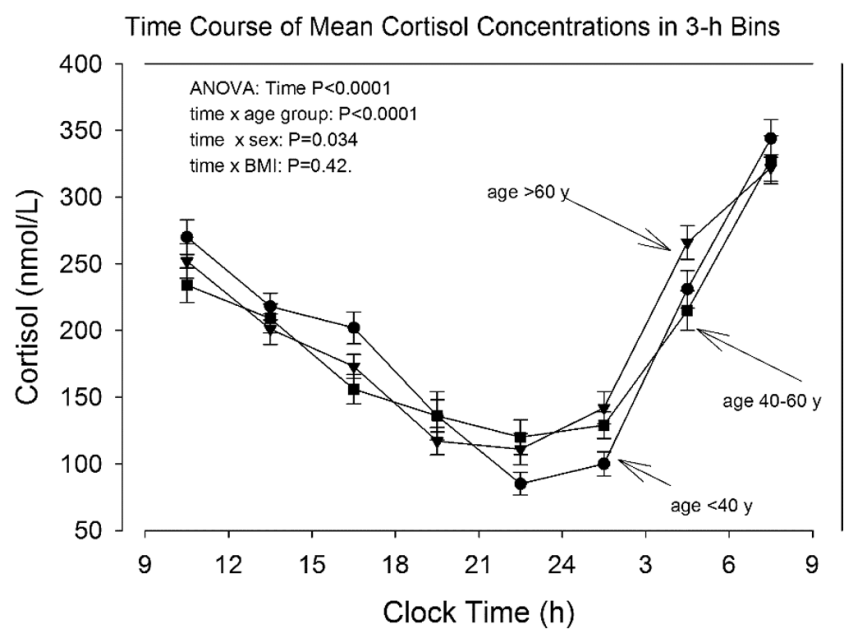

Figure 1

Mean serum cortisol concentrations in 3-h bins and stratified for age. Symbols: circles: subjects younger than 40 years, squares: subjects 40-60 years, triangles: subjects older than 60 years. Additional statistical data are given in Supplementary Table 3. 
assay method. The effect of age was analyzed by linear regression. Calculations were performed using Systat 13 (Systat Software, Inc, San Jose, CA, USA) and Matlab 8.6 (The MathWorks, Inc, Natick, MA, USA). Cosinor analysis was carried out with Sigmaplot 13 (Systat Software, Inc). $P<0.05$ was construed as significant for the overall study. Post hoc multiple comparisons used Sidak's correction.

\section{Results}

Mean 24-h cortisol concentrations were lower in premenopausal women than men of comparable age $(176 \pm 8.2$ vs $217 \pm 9.4 \mathrm{nmol} / \mathrm{L}, P=0.02)$, due to lower cortisol secretory-burst mass $(220 \pm 17.6$ and $266 \pm 16.8 \mathrm{nmol} / \mathrm{L}$, $P=0.05)$. No differences existed after age 50 years.

Mean cortisol concentrations over 24-h and in 3-h bins are shown in Table 2. Cortisol was higher in men than women during daytime only. As shown in 3 different age cohorts in Fig. 1, older individuals had higher cortisol in the late evening and early night (Table 3). Regression analyses delineated positive correlations with age between clock times 21 and $3 \mathrm{~h}$ (Table 4), as illustrated graphically in Fig. 2. The magnitude of the late-day cortisol increment was about $10 \mathrm{nmol} / \mathrm{L}$ per decade.

Results of deconvolution of the 24-h serum cortisol time series are shown in Table 5. Cortisol secretory-burst frequency was slightly higher, whereas cortisol half-life and basal secretion were shorter and lower, respectively, in women than men. Total daily cortisol secretion, secretoryburst mode and pulsing regularity were sex independent. Age and BMI were not significant regression factors for deconvolution parameters (Supplementary Tables 1 and 2, see section on supplementary data given at the end of this article).

Table 3 Cortisol means of $3 \mathrm{~h}$ bins stratified for three age groups.

\begin{tabular}{|c|c|c|c|}
\hline Time bin (h) & $\begin{array}{c}\text { Age } \\
<40 \text { years }(60)\end{array}$ & $\begin{array}{c}\text { Age } \\
40-60 \text { years }(38)\end{array}$ & $\begin{array}{c}\text { Age } \\
>60 \text { years (45) }\end{array}$ \\
\hline $9-12$ & $270 \pm 13$ & $230 \pm 12$ & $252 \pm 13$ \\
\hline $12-15$ & $218 \pm 10$ & $204 \pm 11$ & $201 \pm 12$ \\
\hline $15-18$ & $202 \pm 12$ & $153 \pm 11$ & $173 \pm 8.9$ \\
\hline $18-21$ & $136 \pm 12$ & $130 \pm 18$ & $117 \pm 10$ \\
\hline $21-24$ & $85 \pm 8.6$ & $117 \pm 13$ & $111 \pm 11$ \\
\hline $24-3$ & $100 \pm 9.1$ & $1269 \pm 10$ & $142 \pm 12$ \\
\hline $3-6$ & $231 \pm 14$ & $221 \pm 15$ & $266 \pm 13$ \\
\hline $6-9$ & $344 \pm 17$ & $323 \pm 18$ & $322 \pm 9.8$ \\
\hline
\end{tabular}

GLM procedure for repeat measurements: between subjects: age group: $P=0.43$, sex: $P=0.022$, BMl: $P=0.041$. Within subjects: time (bin): $P<0.0001$, time $\times$ age group: $P<0.0001$, time $\times$ sex: $P=0.034$, time $\times \mathrm{BMI}$ : $P=0.42$.
Table 4 Linear regressions of mean cortisol and age.

\begin{tabular}{|c|c|c|c|c|}
\hline Time bin (h) & $\boldsymbol{R}$ & $\begin{array}{l}\text { Regression } \\
\text { slope } \beta\end{array}$ & $P$ value & $\begin{array}{c}P \text { value log- } \\
\text { transformed data }\end{array}$ \\
\hline $9-12$ & 0.114 & $-0.658 \pm 0.485$ & 0.18 & 0.16 \\
\hline $12-15$ & 0.110 & $-0.526 \pm 0.402$ & 0.19 & 0.19 \\
\hline $15-18$ & 0.213 & $-1.093 \pm 0.421$ & 0.011 & 0.029 \\
\hline $18-21$ & 0.104 & $-0.590 \pm 0.475$ & 0.22 & 0.26 \\
\hline $21-24$ & 0.199 & $0.952 \pm 0.394$ & 0.017 & 0.001 \\
\hline $24-3$ & 0.249 & $1.152 \pm 0.405$ & 0.003 & 0.001 \\
\hline $3-6$ & 0.143 & $0.912 \pm 0.530$ & 0.087 & 0.032 \\
\hline $6-9$ & 0.07 & $-0.475 \pm 0.573$ & 0.41 & 0.77 \\
\hline
\end{tabular}

The regression slopes are shown as mean \pm S.D.

Cosinor analysis (Fig. 3) showed an acrophase advance (earlier clock time) of $0.40 \mathrm{~h}$ ( $24 \mathrm{~min}$ ) per decade. Neither mesor nor amplitude was age dependent (both $P>0.05$ ). As an simple model-free measure of diurnal amplitude, the difference of 3-h cortisol means in the morning (9-12h) and nighttime (21-24h) decreased with age with $R=0.28$, $P=0.001$, and $\beta=1.60 \pm 0.48$.

Cortisol ApEn over $24 \mathrm{~h}$ was $0.921 \pm 0.024$ in men, and $1.019 \pm 0.026$ in women $(P<0.0001)$. In premenopausal women, ApEn was 1.048 \pm 0.025 and higher than men age $<50$ years: $0.933 \pm 0.023, P=0.001$, but not in subjects aged 50 years or more $(0.963 \pm 0.058$ vs $0.909 \pm 0.043, P=0.46)$. There was no BMI effect. Cortisol was higher with age; viz., borderline in the DiaSorin assay $(P=0.06)$, but $P=0.017$ for the Roche assay and $P=0.004$ for the Immulite assay. The time pattern of cortisol ApEn variation across the 3-h bins is illustrated in Fig. 4, showing higher daytime than nighttime values $(P<0.0001)$ and dependency on sex $(P<0.0001)$.

The influence of cortisol assay was analyzed further. The DiaSorin assay group comprised 89 subjects, the Immulite 2000-assay group 34 subjects and the Roche E170 assay group 20 subjects. For the 3 assays, median 24-h cortisol concentrations (145 samples) were $161 \pm 6.1$, $167 \pm 10.5$ and $185 \pm 11.5 \mathrm{nmol} / \mathrm{L}$, respectively. In the GLM procedure, assay type was not significant $(P=0.33)$.

By GLM analysis, age and BMI did not influence 24-h mean cortisol concentrations ( $P$ values 0.11 and 0.36 ), whereas sex did with median levels in men of $176 \pm 6.4$ vs $146 \pm 7.1 \mathrm{nmol} / \mathrm{L}$ in women $(P=0.005)$.

GLM analysis of deconvolution parameters (Table 6) revealed no effects of cortisol assay, age, BMI or sex.

As a sensitivity analysis, leaving out the data obtained in the Roche E170 or Immulite, or both, assays did not alter conclusions.

Progressively reducing sampling intensity affected estimates of mean 24-h cortisol concentration, as portrayed in Fig. 5. The squared correlation coefficient

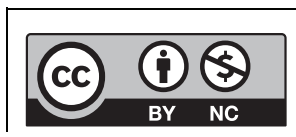

This work is licensed under a Creative Commons Attribution-NonCommercial 4.0 International License. 
Regression of Mean Serum Cortisol Concentrations between $2100 \mathrm{~h}$ and $\mathrm{MN}$ on Age

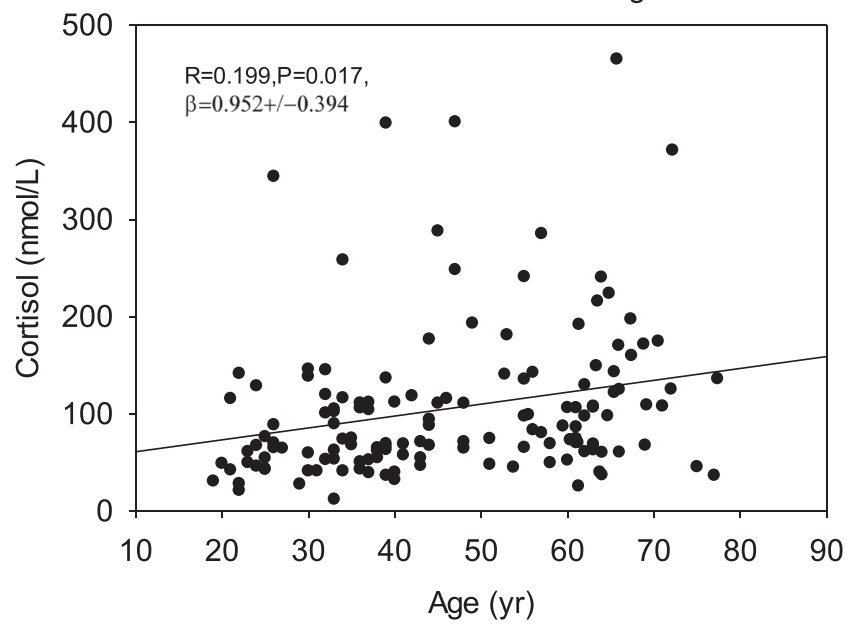

Regression of Mean Serum Cortisol Concentrations between $\mathrm{MN}$ and $0300 \mathrm{~h}$ on Age

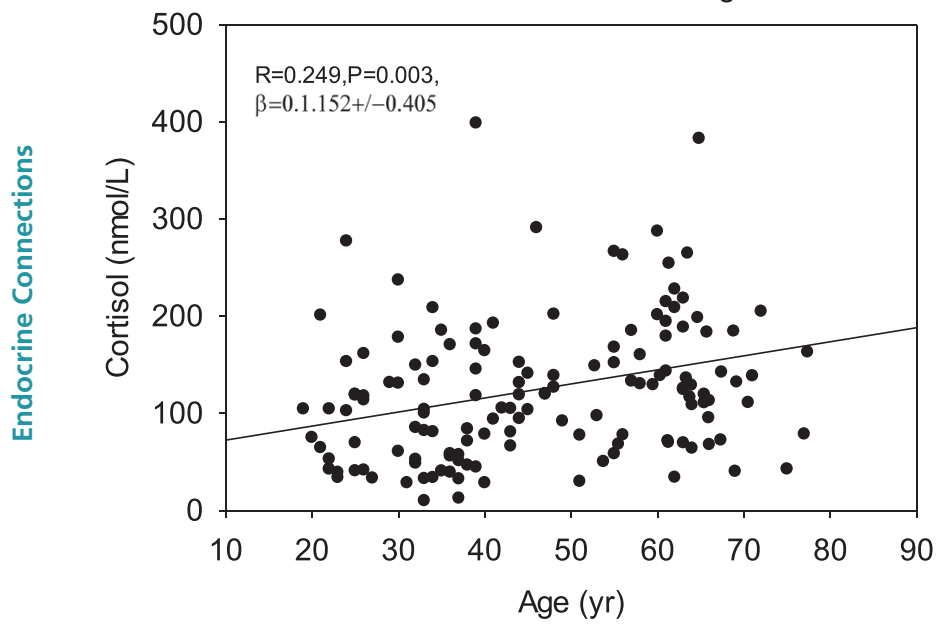

Figure 2

Linear regressions of mean cortisol (3-h) concentrations on age in the clock time intervals of 21-24h (upper panel) and 24-3h (lower panel).

decreased from 0.998 (20 vs 10-min sampling) to 0.91 (120 vs 20 -min sampling). Means of two samples per $24 \mathrm{~h}$, when taken $12 \mathrm{~h}$ apart, yielded $R^{2}=0.51$, thus explaining $51 \%$ of the variability.

\section{Discussion}

This study quantifies simple and complex measures of cortisol secretion with respect to sex, age and BMI utilizing 10-min sampling for $24 \mathrm{~h}$ in 143 adults ages 19-78 years and validated analytical measures of putative feedback control (ApEn), pulsatile and nonpulsatile secretion (deconvolution) and diurnal rhythmicity (Cosinor analysis). Total 24-h cortisol secretion estimated

$\begin{array}{lr}\text { http://www.endocrineconnections.org } & \text { ○ } 2017 \text { The authors } \\ \text { DOI: } 10.1530 / \text { EC-17-0160 } & \text { Published by Bioscientifica Ltd }\end{array}$

Table 5 Deconvolution analysis of serum cortisol profiles in 143 healthy volunteers.

\begin{tabular}{|c|c|c|c|}
\hline Parameter & Men (79) & Women (64) & $P$ value \\
\hline Pulse frequency (\#/24h) & $16.4 \pm 0.5$ & $18.5 \pm 0.6$ & 0.005 \\
\hline Slow half-life (min) & $64.4 \pm 1.0$ & $57.0 \pm 1.3$ & 0.008 \\
\hline Mode day (min) & $11.2 \pm 0.8$ & $14.5 \pm 1.3$ & 0.054 \\
\hline Mode night (min) & $12.1 \pm 0.7$ & $14.5 \pm 1.2$ & 0.19 \\
\hline $\begin{array}{l}\text { Basal secretion } \\
\text { (nmol/L/24h) }\end{array}$ & $903 \pm 66$ & $786 \pm 94$ & 0.007 \\
\hline $\begin{array}{l}\text { Pulsatile secretion } \\
(\mathrm{nmol} / \mathrm{L} / 24 \mathrm{~h})\end{array}$ & $4011 \pm 158$ & $4950 \pm 243$ & 0.68 \\
\hline $\begin{array}{l}\text { Total secretion } \\
(\mathrm{nmol} / \mathrm{L} / 24 \mathrm{~h})\end{array}$ & $4915 \pm 195$ & $4954 \pm 243$ & 0.92 \\
\hline $\begin{array}{l}\text { Mean pulse mass } \\
(\mathrm{nmol} / \mathrm{L})\end{array}$ & $253 \pm 10$ & $236 \pm 13$ & 0.17 \\
\hline Weibull lambda (\#/24h) & $15.7 \pm 0.4$ & 16.9 & 0.09 \\
\hline $\begin{array}{l}\text { Weibull gamma } \\
\text { (dimensionless) }\end{array}$ & $1.816 \pm 0.046$ & $1.846 \pm 0.051$ & 0.59 \\
\hline ApEn (dimensionless) & $0.921 \pm 0.024$ & $1.019 \pm 0.026$ & 0.007 \\
\hline
\end{tabular}

Data are shown as mean and S.E.M. The two-tailed Student's $t$-test was used for statistical comparisons on logarithmically transformed data.

by deconvolution of intensively sampled profiles was independent of sex, age and BMI. In subanalyses, cortisol dynamics exhibited a higher pulse frequency, shorter half-life and lower basal secretion in women than men. Nycthemeral variations differed by sex and age, with higher mean cortisol in the daytime in men than women, and in late evening and early night in older than young subjects. This result seems to be at variance with a study in which cortisol levels were stimulated with low-dose and high-dose ACTH and by glucagon administration in 55 healthy individuals (38).

\section{Acrophase of Cortisol versus Age}

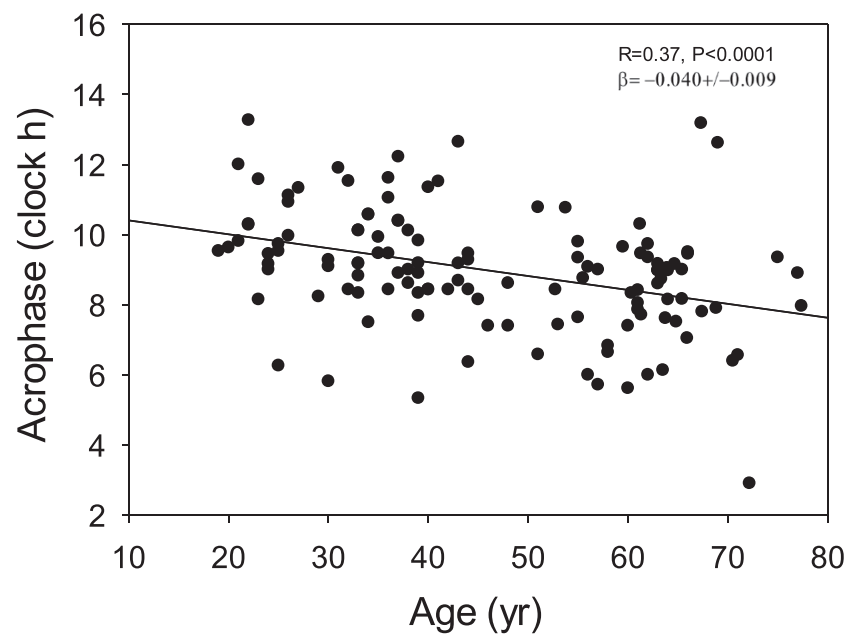

Figure 3

Relation between time of cortisol acrophase (maximal Cosinor-estimated 24-h concentration) and age. The apparent advance (earlier timing) with age was $24 \mathrm{~min}$ per decade. 
Cortisol ApEn across 24-H Time Series.

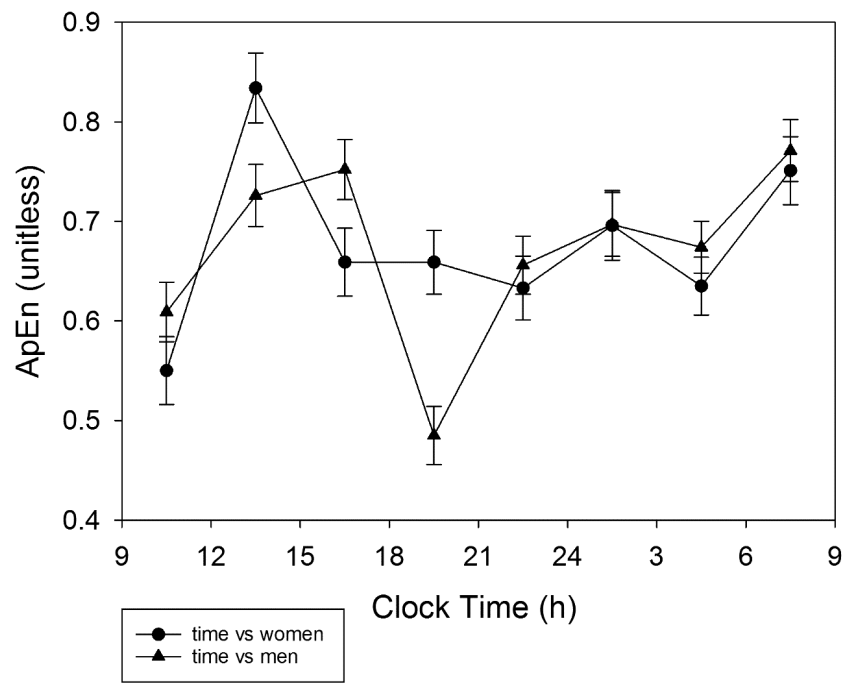

Figure 4

Approximate entropy (ApEn) of cortisol concentrations in men and women, segmented in 3-h time bins. During the morning and afternoon, ApEn was higher than in the remaining part of the diurnal cycle.

In this limited study BMI, age and sex were not significant variables. However, acute stimulation tests are strictly nonphysiological, and therefore, any comparison is hampered by these considerations. Moreover, for a fair comparison between such studies dose-response curves are required, but such expensive and labor-intensive studies are far beyond the clinical requirements.

ApEn was higher in women than men, higher in the daytime and independent of aging. The nycthemeral maximum (acrophase) was advanced to an earlier clock time by $40 \mathrm{~min} /$ decade and increased by about $10 \mathrm{nmol} / \mathrm{L} /$ decade.

HPA axis activity ostensibly varies with age, sex, BMI, socioeconomic status, chronic illness, stress, psychiatric disorders and sleep disruption $(1,13,18)$. Comparisons among studies, however, are hampered by different experimental designs, including number and spacing of blood samples, salivary samples or urinary excretion of cortisol and/or cortisol metabolites, size of groups, sex, BMI and age distributions. Blood sampling intensity in studies in which age was related to mean serum cortisol levels varied from 4 to 72 samples/24h. The study of 145 samples/24h could not demonstrate age effects on mean (median) cortisol concentrations or distinct cortisol secretion parameters. Three studies reported an increase in cortisol area under the curve $(5,6,13)$, a fourth study a decrease in mean 24-h cortisol (10), and a fifth and sixth no influence of age $(10,11,27)$. The present approach differs by using 10-min sampling, 24-h data, a larger cohort of subjects, and all three of deconvolution, Cosinor and ApEn analyses. Nonetheless, the age range of 19-78 years, like most other studies, limits conclusions in the very old.

Cortisol's regulation was examined further using consecutive 3 -h time windows over $24 \mathrm{~h}$, since higher cortisol in the late evening-early night quiescent period has been associated with sleep disorders and the metabolic syndrome $(7,28)$. By cohort comparisons, two studies found higher cortisol concentrations in older than young subjects in the quiescent period $(6,13)$, whereas one study did not (12). The present analysis across continuous ages delineates a late-day rise in mean cortisol concentrations of $10 \mathrm{nmol} / \mathrm{L} /$ decade. This effect could be due to diminished feedback of cortisol on pituitary corticotropes and/or brain CRH/AVP-secreting neurons $(29,30)$ and may have health implications. Thus, the Leiden Longevity Study of 303 subjects disclosed lower salivary cortisol in the late evening in offspring of healthy nonagenarian siblings compared to their partners (8). In another study, hydrocortisone administration during the quiescent phase in metyrapone-clamped volunteers reduced insulin sensitivity and glucose tolerance (31). However, in this study, serum cortisol levels reached about $800 \mathrm{nmol} / \mathrm{L}$, exceeding physiological levels. Conversely, continuous sc infusion of cortisol in Addison's patients

Table 6 Significance values of deconvolution parameters in the GLM procedure of serum cortisol profiles in 143 healthy subjects.

\begin{tabular}{|c|c|c|c|c|}
\hline & $\begin{array}{c}\text { Pulse } \\
\text { frequency }\end{array}$ & $\begin{array}{c}\text { Slow } \\
\text { half-life }\end{array}$ & $\begin{array}{l}\text { Mode } \\
\text { day }\end{array}$ & $\begin{array}{l}\text { Mode } \\
\text { night }\end{array}$ \\
\hline Sex & 0.27 & 0.21 & 0.42 & 0.27 \\
\hline Assay & 0.17 & 0.87 & 0.78 & 0.27 \\
\hline BMI & 0.77 & 0.91 & 0.37 & 0.83 \\
\hline Age & 0.81 & 0.51 & 0.86 & 0.39 \\
\hline Age $\times$ assay & 0.56 & 0.86 & 0.98 & 0.28 \\
\hline \multicolumn{5}{|c|}{$\begin{array}{l}\text { The used cortisol assays were DiaSorin RIA, First generation of the Roche } \mathrm{E} \\
\text { MPM, mean pulse mass. }\end{array}$} \\
\hline
\end{tabular}

\begin{tabular}{l|}
$\begin{array}{c}\text { Basal } \\
\text { secretion }\end{array}$ \\
\hline 0.27 \\
0.578 \\
0.69 \\
0.16 \\
0.51 \\
\hline
\end{tabular}

\begin{tabular}{c}
$\begin{array}{c}\text { Pulsatile } \\
\text { secretion }\end{array}$ \\
\hline 0.46 \\
0.377 \\
0.14 \\
0.68 \\
0.67
\end{tabular}

\begin{tabular}{c}
$\begin{array}{c}\text { Total } \\
\text { secretion }\end{array}$ \\
\hline 0.30 \\
0.37 \\
0.16 \\
0.38 \\
0.52 \\
\hline
\end{tabular}

\begin{tabular}{lllll}
\hline MPM & & Lambda & & Gamma \\
\cline { 1 - 1 } 0.11 & & 0.32 & & 0.66 \\
0.92 & & 0.47 & & 0.55 \\
0.41 & & 0.78 & & 0.51 \\
0.98 & & 0.84 & & 0.42 \\
0.92 & & 0.60 & & 0.51
\end{tabular}

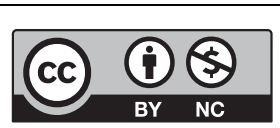

This work is licensed under a Creative Commons Attribution-NonCommercial 4.0 International License. 
Squared Correlations between 144 Samples-based Mean and Decreasing Number of Samples.

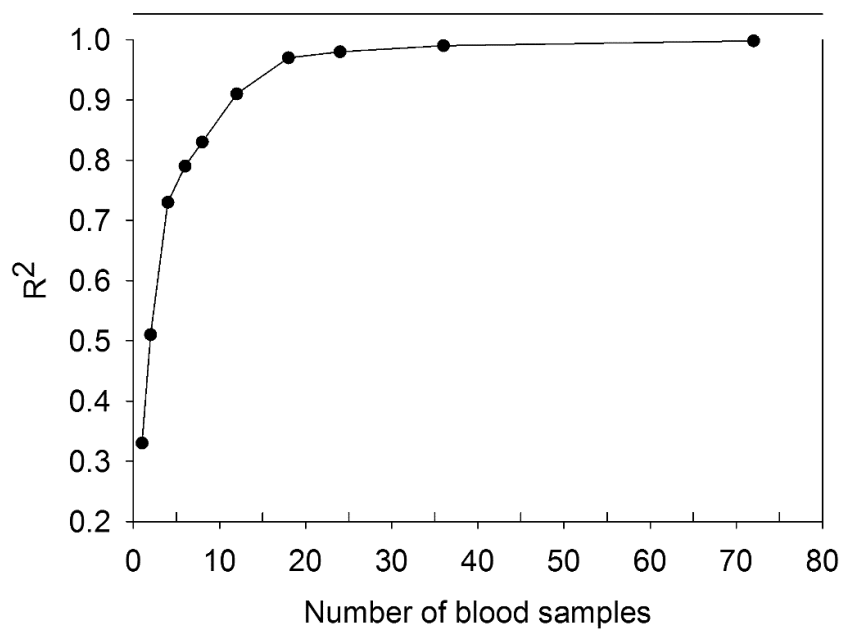

Figure 5

Relationship between the mean cortisol concentration determined from decreasing sampling interval ( $x$-axis) and mean cortisol level based on 144 samples taken at $10 \mathrm{~min}$ intervals for $24 \mathrm{~h}$. The squared correlation coefficients determined by linear regressions are shown on the $y$-axis.

was sufficient to prevent the nocturnal glucose decrease (32). Direct experimental data are needed on the impact of glucocorticoid levels in this time window over days or weeks on other health indices, such as blood pressure, abdominal adiposity, diabetes control and systemic inflammation.

Few studies have assessed whether 24-h cortisol secretion is sex dependent or estradiol dependent. One small isotope dilution study in 7 men and 7 women under age 40 years estimated nearly 50\% lower cortisol production in women than men (14). Another study reported lower mean 24-h cortisol concentrations in women than men, supporting the present findings in volunteers under age 50 years (13). Using 24-h sampling, we could show that cortisol concentrations were higher in men only during daytime. This insight would agree with higher single-morning total and free cortisol levels reported in men earlier (33).

The present analysis revealed that cortisol secretoryburst mass is smaller and half-life shorter in premenopausal women than men under age 50 years. Because of a higher pulse frequency, no sex difference emerged for total cortisol secretion. Although the role of (premenopausal) estrogen is not so clear (18), in older adults shortterm administration of estradiol, but not testosterone, heightens cortisol-mediated inhibition of pulsatile ACTH secretion (15). In principle, therefore, an estrogenic milieu could lower mean cortisol in premenopausal women.
Higher BMI is associated with attenuated 24-h GH secretion, and a more subtly increased 24 -h TSH, prolactin and ACTH secretion $(4,21,34,35)$. Although urinary levels of cortisol and its metabolites rise in obese individuals, this is not true when normalized for fat mass or BMI. Concomitantly, serum cortisol levels remain unchanged or even decrease, due to increases in distribution volume and metabolic clearance (35). In accordance with these reports, the current analysis found no significant effect of BMI along or in combination with sex and age on 24-h cortisol concentrations or multiple dynamic measures of cortisol secretion.

Cosinor analysis is a widely used tool to quantify 24-h rhythms of adrenal steroids, sex steroids, TSH, GH, LH, FSH, prolactin and deep body temperature. Herein, we found, consistent with published data, an advance in the acrophase of cortisol, defined by cortisol's peaking about $0.4 \mathrm{~h}$ earlier in the day per decade of age $(6,10$, 11). Although Cosinor analysis detected no effect of age on either the cortisol mesor or amplitude, a modelfree approach revealed an amplitude decrease of about $16 \mathrm{nmol} / \mathrm{L}$ per decade age. As suggested earlier (22), sensitivity of the Cosinor method is reduced when the nadir and acrophase are not $12 \mathrm{~h}$ apart.

ApEn is a measure of serial regularity, reflecting the balance between feedforward and feedback strength (24). Our new finding that cortisol ApEn is higher in women than men is theoretically compatible with inferred increased feedforward of CRH, AVP and ACTH and diminished feedback by dexamethasone and cortisol (16, 17, 29, 30, 36, 37). Cortisol ApEn was age dependent only in sex-specific evaluations. Recent data on ApEn of GH, TSH and prolactin rises with age in both sexes, consistent with altered feedforward/feedback control. At this time, we have no explanation for possible assay dependency of cortisol ApEn.

Intensive 24-h hormone studies are expensive and therefore rare. Only two large studies on cortisol profiles are available, one by van Cauter and coworkers (13) and the present one. They differ in design, i.e., sampling interval and used analytical techniques. Aside from applying deconvolution of the profiles we used a model-free approach to quantitate particular time intervals instead of the best fit curve based on periodogram calculations. Both studies had, not unexpectedly, similarities but also differences. In the present study, mean 24-h cortisol concentration was independent of sex, age and BMI, and this result was corroborated by deconvolution, while the other study showed an age-dependent increase. On the other hand, both studies showed age-dependent

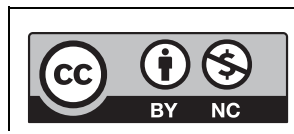

This work is licensed under a Creative Commons Attribution-NonCommercial 4.0 International License. 
concentration increases in the quiescent period of the 24-h cycle. In the present study, an age-dependent advance shift of the acrophase was found, but not in the study by van Cauter, although they described an advance shift in the timing of the onset of the diurnal concentration increase. Thus summarizing, differences and similarities between both studies exist, partly attributable to different analytical approaches.

For clinicians, this study has only restricted relevance. A major point to realize is that a single-morning cortisol specimen has very limited predictability of the 24-h secretion. Furthermore, mean 3-h morning cortisol levels are higher in men than women, and there is an age-related increase in levels during the quiescent hours in the late evening and early night. For diagnostic considerations, these subtle physiological adaptations are not relevant in diagnosing major endocrine disruptions such as Cushing's disease, Cushing's syndrome and primary and secondary adrenal failure. For these disorders, Endocrine Societies have formulated clear guidelines. The most challenging condition is the differential diagnosis between ectopic ACTH secretion and pituitary-dependent hypercortisolemia, when imaging and pituitary blood sampling studies are equivocal. Unfortunately, for such cases, no data are available whether extensive evaluation of plasma ACTH-cortisol profiles is helpful in making a distinction.

A potential problem inherent in retrospective studies is that different hormone assays are used. Extensive statistical GLM analyses, wherein assay type was a potential dependent variable, did not reveal statistically significant assay effects. This was confirmed by sensitivity analyses, which entailed removing one assay at a time. A similar issue exists in other large retrospective analyses, such as with data from 9 different institutions and thus different cortisol assays (13).

When the clinical goal is an accurate estimate of the mean 24-h cortisol concentration 10-min sampling is not required. An accurate $24-\mathrm{h}$ mean value can be obtained from as few as 4 equally distributed (e.g. every $6 \mathrm{~h}$ ) blood samples.

Limitations of the present study include the needs to study younger adults ( $\leq$ age 18 years) and quite old individuals ( $\geq$ age 80 years); verify 24 -h rhythms by 3-5 days of 10-min sampling; estimate body composition beyond BMI per se; quantify free cortisol concentrations and/or CBG levels and conduct similar analyses as an additional function of season. In addition, the use of a single high-sensitivity immunological cortisol assay or HPLC-tandem MS-MS is preferable.
In summary, healthy adults maintain consistent 24-h cortisol concentrations independently of BMI. In relation to age, the cortisol acrophase advances about $24 \mathrm{~min} /$ decade and late evening and early night cortisol increases about $10 \mathrm{nmol} / \mathrm{L} /$ decade. Sex differences in mean cortisol concentrations operate during the daytime in the premenopausal age range of $<50$ year. Cortisol ApEn is higher in the day than night and in premenopausal women than men, denoting less cortisol feedback control. ApEn was BMI and age independent. The collective outcomes should provide a better framework for interpreting cortisol physiology and pathophysiology in health and disease.

\section{Supplementary data}

This is linked to the online version of the paper at http://dx.doi.org/10.1530/ EC-17-0160.

\section{Declaration of interest}

The authors declare that there is no conflict of interest that could be perceived as prejudicing the impartiality of the research reported.

\section{Funding}

Supported in part via R01 AG019695, R01 DK073148, R01 AG029362, R01 AG031763 and P30 DK050456 (Metabolic Studies Core of the Minnesota Obesity Center) from the National Institutes of Health (Bethesda, MD, USA). Matlab versions of ApEn and deconvolution methodology are available from Veldhuis.johannes@mayo.edu. The project described was supported by UL1 TR000135 from the National Center for Advancing Translational Sciences (NCATS) and 60NANB10D005Z from the National Institute of Standards and Technology. Contents are solely the responsibility of the authors and do not necessarily represent the official views of any federal institution.

\section{Author contribution statement}

$F R$, J D V and P T helped in conception/design of research. D V H, P T, R Y and F R performed experiments. F R and J D V analyzed the data. F R and $J \mathrm{D} V$ helped in manuscript preparation. All authors approved the final manuscript.

\section{Acknowledgments}

The authors thank Jill Smith for support of manuscript preparation.

\section{References}

1 Seeman T, Epel E, Gruenewald T, Karlamangla A \& McEwen BS Socio-economic differentials in peripheral biology: cumulative allostatic load. Annals of the New York Academy of Sciences 20101186 223-239. (doi:10.1111/j.1749-6632.2009.05341.x)

2 Roelfsema F, Pincus SM \& Veldhuis JD. Patients with Cushing's disease secrete adrenocorticotropin and cortisol jointly more asynchronously than healthy subjects. Journal of Clinical Endocrinology and Metabolism 199883 688-692. (doi:10.1210/jc.83.2.688)

3 Jansen SW, Roelfsema F, Akintola AA, Oei NY, Cobbaert CM, Ballieux BE, van der Grond J, Westendorp RG, Pijl H \& van Heemst D.

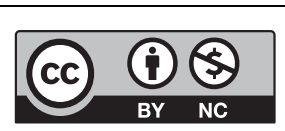

This work is licensed under a Creative Commons Attribution-NonCommercial 4.0 International License. 
Characterization of the hypothalamic-pituitary-adrenal-axis in familial longevity under resting conditions. PLOS ONE $2015 \mathbf{1 0}$ e0133119. (doi:10.1371/journal.pone.0133119)

4 Roelfsema F, Pijl H, Keenan DM \& Veldhuis JD. Diminished adrenal sensitivity and adrenocorticotropin efficacy in obese premenopausal women. European Journal of Endocrinology 2012167 633-642. (doi:10.1530/EJE-12-0592)

5 Purnell JQ, Brandon DD, Isabelle LM, Loriaux DL \& Samuels MH. Association of 24-hour cortisol production rates, cortisol-binding globulin, and plasma-free cortisol levels with body composition, leptin levels, and aging in adult men and women. Journal of Clinical Endocrinology and Metabolism 200489 281-287. (doi:10.1210/jc.2003030440)

6 Deuschle M, Gotthardt U, Schweiger U, Weber B, Korner A, Schmider J, Standhardt H, Lammers CH \& Heuser I. With aging in humans the activity of the hypothalamus-pituitary-adrenal system increases and its diurnal amplitude flattens. Life Science $1997 \mathbf{6 1}$ 2239-2246. (doi:10.1016/S0024-3205(97)00926-0)

7 Vgontzas AN, Zoumakis M, Bixler EO, Lin HM, Prolo P, Vela-Bueno A, Kales A \& Chrousos GP. Impaired nighttime sleep in healthy old versus young adults is associated with elevated plasma interleukin-6 and cortisol levels: physiologic and therapeutic implications. Journal of Clinical Endocrinology and Metabolism 200388 2087-2095. (doi:10.1210/jc.2002-021176)

8 Noordam R, Jansen SW, Akintola AA, Oei NY, Maier AB, Pijl H, Slagboom PE, Westendorp RG, van der Grond J, de Craen AJ, et al. Familial longevity is marked by lower diurnal salivary cortisol levels: the Leiden Longevity Study. PLoS ONE 20127 e31166. (doi:10.1371/ journal.pone.0031166)

9 Aziz NA, Pijl H, Frolich M, van der Graaf AW, Roelfsema F \& Roos RA. Increased hypothalamic-pituitary-adrenal axis activity in Huntington's disease. Journal of Clinical Endocrinology and Metabolism 200994 1223-1228. (doi:10.1210/jc.2008-2543)

10 Sherman B, Wysham C \& Pfohl B. Age-related changes in the circadian rhythm of plasma cortisol in man. Journal of Clinical Endocrinology and Metabolism 198561 439-443. (doi:10.1210/ jcem-61-3-439)

11 Waltman C, Blackman MR, Chrousos GP, Riemann C \& Harman SM. Spontaneous and glucocorticoid-inhibited adrenocorticotropin hormone and cortisol secretion are similar in healthy young and old men. Journal of Clinical Endocrinology and Metabolism 199173 495-502. (doi:10.1210/jcem-73-3-495)

12 Ferrari E, Cravello L, Muzzoni B, Casarotti D, Paltro M, Solerte SB, Fioravanti M, Cuzzoni G, Pontiggia B \& Magri F. Age-related changes of the hypothalamic-pituitary-adrenal axis: pathophysiological correlates. European Journal of Endocrinology 2001144 319-329. (doi:10.1530/eje.0.1440319)

13 Van Cauter E, Leproult R \& Kupfer DJ. Effects of gender and age on the levels and circadian rhythmicity of plasma cortisol. Journal of Clinical Endocrinology and Metabolism 199681 2468-2473. (doi:10.1210/jc.81.7.2468)

14 Vierhapper H, Nowotny P \& Waldhausl W. Sex-specific differences in cortisol production rates in humans. Metabolism 199847 974-976. (doi:10.1016/S0026-0495(98)90353-5)

15 Sharma AN, Aoun P, Wigham JR, Weist SM \& Veldhuis JD. Estradiol, but not testosterone, heightens cortisol-mediated negative feedback on pulsatile ACTH secretion and ACTH approximate entropy in unstressed older men and women. American Journal of Physiology: Regulatory Integrative and Comparative Physiology 2014306 R627-R635. (doi:10.1152/ajpregu.00551.2013)

16 Parker CR Jr, Slayden SM, Azziz R, Crabbe SL, Hines GA, Boots LR \& Bae S. Effects of aging on adrenal function in the human: responsiveness and sensitivity of adrenal androgens and cortisol to adrenocorticotropin in premenopausal and postmenopausal women. Journal of Clinical Endocrinology and Metabolism 200085 48-54. (doi:10.1210/jc.85.1.48)
17 Greenspan SL, Rowe JW, Maitland LA, McAloon-Dyke M \& Elahi D. The pituitary-adrenal glucocorticoid response is altered by gender and disease. Journal of Gerontology 199348 M72-M77. (doi:10.1093/ geronj/48.3.M72)

18 Veldhuis JD, Sharma A \& Roelfsema F. Age-dependent and genderdependent regulation of hypothalamic-adrenocorticotropic-adrenal axis. Endocrinology Metabolism Clinics of North America 201342 201-225. (doi:10.1016/j.ecl.2013.02.002)

19 Keenan DM \& Veldhuis JD. Divergent gonadotropin-gonadal dose-responsive coupling in healthy young and aging men. American Journal of Physiology 2004286 R381-R389. (doi:10.1152/ ajpregu.00376.2003)

20 Keenan DM, Roelfsema F, Biermasz N \& Veldhuis JD. Physiological control of pituitary hormone secretory-burst mass, frequency and waveform: a statistical formulation and analysis. American Journal of Physiology 2003285 R664-R673. (doi:10.1152/ajpregu.00195.2003)

21 Kok P, Roelfsema F, Frolich M, Meinders AE \& Pijl H. Spontaneous diurnal thyrotropin secretion is enhanced in proportion to circulating leptin in obese premenopausal women. Journal of Clinical Endocrinology and Metabolism 200590 6185-6191. (doi:10.1210/ jc.2005-0003)

22 Veldhuis JD, Keenan DM \& Pincus SM. Motivations and methods for analyzing pulsatile hormone secretion. Endocrine Reviews 200829 823-864. (doi:10.1210/er.2008-0005)

23 Liu PY, Keenan DM, Kok P, Padmanabhan V, O’Byrne KT \& Veldhuis JD. Sensitivity and specificity of pulse detection using a new deconvolution method. American Journal of Physiology: Endocrinology and Metabolism 2009297 E538-E544. (doi:10.1152/ ajpendo.00071.2009)

24 Veldhuis JD \& Pincus SM. Orderliness of hormone release patterns: a complementary measure to conventional pulsatile and circadian analyses. European Journal of Endocrinology 1998138 358-362. (doi:10.1530/eje.0.1380358)

25 Van den Berg G, Frolich M, Veldhuis JD \& Roelfsema F. Growth hormone secretion in recently operated acromegalic patients. Journal of Clinical Endocrinology and Metabolism 199479 1706-1715. (doi:10.1210/jc.79.6.1706)

26 Groote Veldman R, van den Berg G, Pincus SM, Frolich M, Veldhuis JD $\&$ Roelfsema F. Increased episodic release and disorderliness of prolactin secretion in both micro- and macroprolactinomas. European Journal of Endocrinology 1999140 192-200. (doi:10.1530/eje.0.1400192)

27 van Coevorden A, Mockel J, Laurent E, Kerkhofs M, L'HermiteBaleriaux M, Decoster C, Neve P \& Van Cauter E. Neuroendocrine rhythms and sleep in aging men. American Journal of Physiology 1991 260 E651-E661.

28 Guyon A, Balbo M, Morselli LL, Tasali E, Leproult R, L'HermiteBaleriaux M, Van CE \& Spiegel K. Adverse effects of two nights of sleep restriction on the hypothalamic-pituitary-adrenal axis in healthy men. Journal of Clinical Endocrinology and Metabolism 201499 2861-2868. (doi:10.1210/jc.2013-4254)

29 Boscaro M, Paoletta A, Scarpa E, Barzon L, Fusaro P, Fallo F \& Sonino N. Age-related changes in glucocorticoid fast feedback inhibition of adrenocorticotropin in man. Journal of Clinical Endocrinology and Metabolism 199883 1380-1383. (doi:10.1210/jc.83.4.1380)

30 Wilkinson CW, Petrie EC, Murray SR, Colasurdo EA, Raskind MA \& Peskind ER. Human glucocorticoid feedback inhibition is reduced in older individuals: evening study. Journal of Clinical Endocrinology and Metabolism 200186 545-550. (doi:10.1210/jc.86.2.545)

31 Plat L, Leproult R, L'Hermite-Baleriaux M, Fery F, Mockel J, Polonsky KS \& Van Cauter E. Metabolic effects of short-term elevations of plasma cortisol are more pronounced in the evening than in the morning. Journal of Clinical Endocrinology and Metabolism 199984 3082-3092. (doi:10.1210/jc.84.9.3082)

32 Bjornsdottir S, Oksnes M, Isaksson M, Methlie P, Nilsen RM, Hustad S, Kampe O, Hulting AL, Husebye ES, Lovas K, et al. Circadian hormone profiles and insulin sensitivity in patients 
with Addison's disease: a comparison of continuous subcutaneous hydrocortisone infusion with conventional glucocorticoid replacement therapy. Clinical Endocrinology 201583 28-35. (doi:10.1111/cen.12670)

33 Sofer Y, Osher E, Limor R, Shefer G, Marcus Y, Shapira I, Tordjman K, Greenman Y, Berliner S \& Stern N. Gender determines serum free cortisol: higher levels in men. Endocrine Practices 201622 1415-1421. (doi:10.4158/EP161370.OR)

34 Roelfsema F \& Veldhuis JD. Growth-hormone dynamics in healthy adults are related to age and gender, and strongly dependent on body mass index. Neuroendocrinology 2016103 335-344. (doi:10.1159/000438904)

35 Roelfsema F, Pereira AM \& Veldhuis JD. Impact of adiposity and fat distribution on the dynamics of adrenocorticotropin and cortisol rhythms. Current Obesity Reports 20143 387-395. (doi:10.1007/s13679-014-0118-7)
36 Liu JH, Rasmussen DD, Rivier J, Vale W \& Yen SS. Pituitary responses to synthetic corticotropin-releasing hormone: absence of modulatory effects by estrogen and progestin. American Journal of Obstetrics and Gynecology 1987157 1387-1391. (doi:10.1016/S00029378(87)80229-6)

37 Luisi S, Tonetti A, Bernardi F, Casarosa E, Florio P, Monteleone P, Gemignani R, Petraglia F, Luisi M \& Genazzani AR. Effect of acute corticotropin releasing factor on pituitary-adrenocortical responsiveness in elderly women and men. Journal of Endocrinological Investigation 199821 449-453. (doi:10.1007/BF03347325)

38 Karaca Z, Lale A, Tanriverdi F, Kula M, Unluhizarci K \& Kelestimur F. The comparison of low and standard dose ACTH and glucagon stimulation tests in the evaluation of hypothalamo-pituitary-adrenal axis in healthy adults. Pituitary $2011 \mathbf{1 4} 134-140$. (doi:10.1007/ s11102-010-0270-3

Received in final form 19 July 2017

Accepted 31 July 2017

Accepted Preprint published online 31 July 2017 http://www.endocrineconnections.org DOI: 10.1530/EC-17-0160
(C) 2017 The authors Published by Bioscientifica Ltd

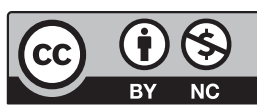

This work is licensed under a Creative Commons Attribution-NonCommercial 4.0 International License. 